\title{
Hierarchies and the Sloshing Bucket: Toward the Unification of Evolutionary Biology
}

\author{
Niles Eldredge
}

Published online: 20 November 2007

(C) Springer Science + Business Media, LLC 2007

\begin{abstract}
Evolutionary biology presents a bewildering array of phenomena to scientists and students alike - ranging from molecules to species and ecosystems; and embracing 3.8 billion years of life's history on earth. Biological systems are arranged hierarchically, with smaller units forming the components of larger systems. The evolutionary hierarchy, based on replication of genetic information and reproduction, is a complex of genes/organisms/demes/species and higher taxa. The ecological hierarchy, based on patterns of matter-energy transfer, is a complex of proteins/organisms/ avatars/local ecosystems/regional ecosystems. All organisms are simultaneously parts of both hierarchical systems. Darwin's original formulation of natural selection maps smoothly onto a diagram where the two hierarchical systems are placed side-by-side. The "sloshing bucket" theory of evolution emerges from empirical cases in biological history mapped onto this dual hierarchy scheme: little phenotypically discernible evolution occurs with minor ecological disturbance; conversely, greatest concentrations of change in evolutionary history follow mass extinctions, themselves based on physical perturbations of global extent. Most evolution occurs in intermediate-level regional "turnovers," when species extinction leads to rapid evolution of new species. Hierarchy theory provides a way of integrating all fields of evolutionary biology into an easily understoodand taught-rubric.
\end{abstract}

N. Eldredge $(\square)$

The American Museum of Natural History,

Central Park West @ 79th St.,

New York, NY 10024, USA

e-mail: epunkeek@amnh.org
Keywords Evolutionary hierarchy · Ecological hierarchy Species $\cdot$ Ecosystems $\cdot$ Sloshing bucket theory of evolution

\section{Introduction}

Evolutionary biology is a notoriously diffuse field of scientific inquiry. It embraces everything from viruses and bacteria through redwoods and leopards, spans nearly 4 billion years and covers the entire globe. It involves phenomena at vastly different scales of time and space: from evanescent molecules to regional ecosystems and species that exist on time scales measured in the millions of years. Scientific study of evolution includes concepts of variation, inheritance, and (starting with Darwin) selection; fossils and the ancient sediments in which they are embedded; patterns of geographic variation and biogeography; species and higher taxa in the framework of systematics (the study of patterns of relationships among species and the classification of life); ecology, developmental biology, and comparative anatomy; and, most recently molecular biology.

Charles Darwin was probably the last person with expertise in virtually all the contemporary disparate fields of knowledge that make up his intellectual brainchild, evolutionary biology. The subsequent exponential growth in knowledge and the fragmentation of fields of study - and hence education and training in these fields - has long since prevented anyone from mastering more than a fraction of the relevant subject matter (although we routinely and rather cavalierly expect secondary school teachers to present a coherent overview of the entire range of topics!). Ideally, every graduate student in evolutionary biology should have at least a passing knowledge of how to: (1) find, excavate, and study a fossil; (2) sample the living 
biota, identify its components, and analyze elements of development, variation, and ecology; and (3) sequence genes and understand how the resultant information dovetails (or not!) with evidence derived from morphology and the fossil record. However, training programs so eclectic and general are a seldom-encountered luxury-with the result that evolution is studied in many different buildings on university campuses, with little or no communication between them.

Since Darwin's death, all evolutionary biologists have agreed that adaptation through natural selection is the core dynamic evolutionary process. Problems only arise when the relatively simple processes of variation, inheritance, and selection are considered not just central and vital, but virtually the sole evolutionary processes of any significance. This assumption-i.e., that heritable variation and selection are not only necessary, but virtually sufficient, to understand how evolution occurs-works fine if you happen to be a population biologist studying variation and selection in the field or in the lab. But if you happen to be a specialist in Paleozoic trilobites (as I have been for many years), such concepts are virtually impossible to apply directly to the data at hand. To speak of "natural selection" with respect to ancient fossils is actually to apply the concept axiomatically-with no way to test directly how selection once worked among those extinct populations and species. No doubt selection shaped the adaptations of my Devonian trilobites every bit as much as we find it does in modern coevolving populations of plants and their insect pollinators; but still there is no practical way actually to study the selection process in long-extinct species, where the fine details of the structure of populational variation are so poorly preserved. And yet Paleozoic trilobites do in fact have much to tell us about the nature of the evolutionary process.

\section{Biological Hierarchies}

There is another way to tease apart the bewildering complexity of biological systems taking part in the evolutionary process - and a way to see how those systems interact, fitting together to yield a more complete, yet easily grasped, understanding of how the evolutionary process actually works to produce the diversity of life through time. The history of biology itself makes it obvious that there are biological systems of different spatiotemporal dimensionsas biology is organized into various nearly discrete subdisciplines: cytology, anatomy, embryology/developmental biology; genomics, "physiological genetics" (Dobzhansky's term - see reference 1), as distinct from population genetics, ecology (itself divided into branches studying everything from small-scale interactions to entire communities and ecosystems of varying scales), systematics, paleontology, and so on. These various biological subdisciplines have an inherent, ontological basis for their existence: they were devised to study systems with their own peculiar spatiotemporal dimensions, with their own origins, histories, and eventual demises, and with internal dynamics that cohere those systems, leading to stability and change through time.

The Genealogical Hierarchy Consider, for example, speciesdefined here as sexually reproducing aggregates of populations sharing a common mate recognition/fertilization system. This definition is a variant of the "biological species concept," associated largely with two of the architects of the "modern synthesis," Theodosius Dobzhansky and Ernst Mayr ${ }^{1}$, although it can be found in the writings of Charles Darwin and many evolutionary biologists who followed. By this definition, species are considered reproductive communities within which sexual reproduction routinely occurs, and beyond which it does not (with some major exceptions, especially in certain groups of plants). The dynamic that keeps species simultaneously bound together, and at the same time separate from other, especially closely related, species is that shared fertilization system. No matter how little or great the gene flow between far-flung populations ("demes" in the context of reproduction), species still cohere over space and through time as long as the fertilization system remains viable in all component demes. Thus species are reproductive communities with spatiotemporal dimensions: they are spread out in space, and form persistent lineages through time.

And fertilization systems diverge-an empirical conclusion from the simple fact that there is more than one species on the planet at any one time. That is, species have origins when lineages diverge. And, inevitably, they have deaths. The vast majority of species that have ever existed are extinct. It was Darwin who finally carried the day with his insistence that if species have deaths we can attribute to natural causes, so must their births be the result of natural - rather than miraculouscauses. Species, like individual organisms, have births, "lifespans," and deaths - an early analogy that Darwin drew in his geology notes in February $1835^{2}$, written in Chile while he was still on the Beagle voyage.

\footnotetext{
${ }^{1}$ Although the "biological species concept" is usually associated with Ernst Mayr [especially Mayr, E. (1942) Systematics and the Origin of Species, Columbia University Press, N.Y.], it was Dobzhansky's earlier book [Dobzhansky. Th. (1937) Genetics and the Origin of Species, Columbia University Press, New York] that first reestablished a reproductive concept of species in evolutionary biology.

2 Darwin's "February 1835" essay is housed along with his other Geology Notes at Cambridge University Library. The essay was transcribed and published in Hodge, M.J.S. (1983). Darwin and the laws of the animate part of the terrestrial system (1835-1837): on the Lyellian origins of his zoonomical explanatory program. Studies in the History of Biology 9:1-106.
} 
Individuals are parts of local breeding populations: demes. Demes are parts of species. In other words, there is a hierarchy of parts and wholes that automatically arises as a byproduct of sexual reproduction. When species split to yield two or more lineages, clusters of related lineages are formed: the higher taxa of the Linnaean hierarchysuch as genera, families, on up through Kingdoms. This is the evolutionary—or, better, "genealogical"—hierarchy, depicted in Fig. 1.

The Ecological Hierarchy But there are other types of larger-scale biological systems, also formed by the interactive behaviors of their component organisms. Consider local ecosystems. John Damuth (Damuth 1985) has made the useful distinction between demes (local reproductive populations of species) and avatars - local populations of organisms of the same species (i.e., "conspecifics") in an "economic" sense. Organisms require energy and nutrient resources to develop, grow, and simply survive. Although much of evolutionary biology has understandably focused on the dynamics of reproduction and its consequences, in reality the vast proportion of an organism's lifespan is given over to economic, rather than reproductive, pursuits. Simply stated, the business of avatars is to survive, thereby providing both a network and context for the business of demes, which is to reproduce.

Conspecific organisms in local avatars - that is, members of the same species in local economic groupsvariably compete or cooperate in the moment-by-moment actions of procuring resources and all the related activities pertaining to sheer survival (such as predator avoidance). And it is their local avatars which form dynamic parts of local ecosystems: although it is individual hawks that eat

\section{Evolutionary Hierarchy}

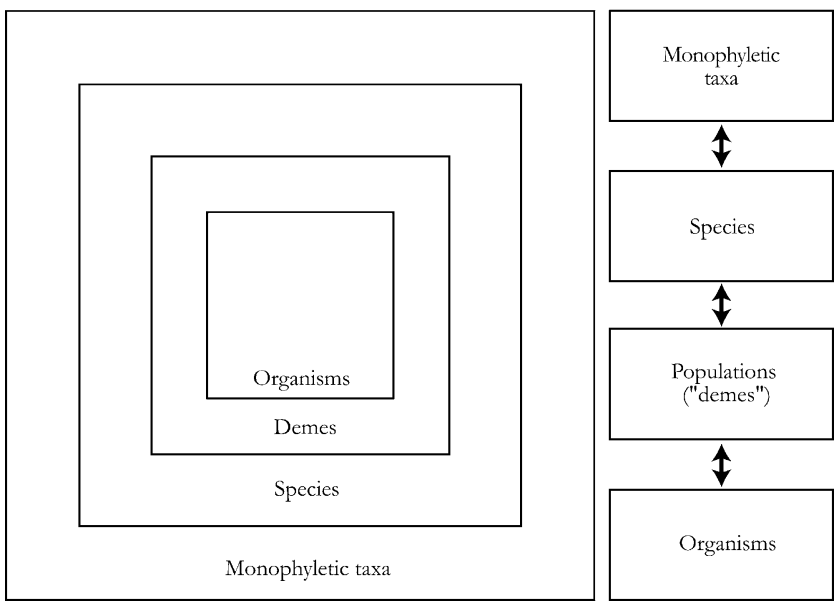

Fig. 1 The evolutionary hierarchy individual squirrels, in reality it is the local population of red-tailed hawks that puts predation pressure on the local eastern gray squirrels (and other prey populations). The role an avatar plays in a local ecosystem is really where the concept of "ecological niche" has its most precise meaning.

Matter and energy flows between avatars of different species, providing the cohesion that keeps local ecosystems together. When local populations of tortoises in the Galapagos are diminished or driven completely extinct, the prickly pear cacti on which they feed suffer (rather than thrive without their major predator) - because seeds of the local Galapagos Opuntia must pass through the gut of a tortoise to germinate.

Although boundaries of local ecosystems are in some cases sharply defined (a stream cutting through a forest, for example), matter/energy flows routinely from system to adjacent system; thus, local ecosystems are interconnected in dynamic ways, forming regional biomes on up through the entire global biotic system - perhaps a useful way of thinking of Lovelock's term "Gaia." This economic-or "ecological"-hierarchy is depicted in Fig. 2.

Thus, an ecologist may study the dynamic interactions of avatars in local ecosystems - such as a stream-without, for example, being concerned with where or how the species supplying those local avatars arose in the first place. Conversely, a systematic paleontologist may study the phylogenetic relationships and speciation patterns and history of a group of related species without paying a great deal of attention to the dynamic properties of the ecosystems where they occur as avatars. This is why ecology and systematics and other branches of evolutionary biology have long had their separate histories.

Yet clearly the two hierarchical systems-genealogical and ecological-must be connected in some way. At the very least, organisms are simultaneously parts of both hierarchies. And the adaptations of organisms - beyond the ones that form the fertilization system itself-are all concerned with the economic needs of organisms. If we place the two hierarchies side-by-side (Fig. 3), we instantly see how Darwin's original concept of natural selection maps onto the diagram. Ongoing reproduction within demes means that the organisms of local avatars are constantly being replenished; as Darwin realized from reading Malthus, more organisms are produced each generation than are needed simply to replace their parents. Hence his "struggle for existence": in terms of our diagram of the two hierarchies, it is those organisms who fare the best economically within the local ecosystems who, as a side effect, will be most successful at reproducing, thus passing along their genetic basis of economic success to the next generation. The status quo is maintained unless and until conditions change, and (provided the needed genetic variation is present), selection modifies adaptations. 


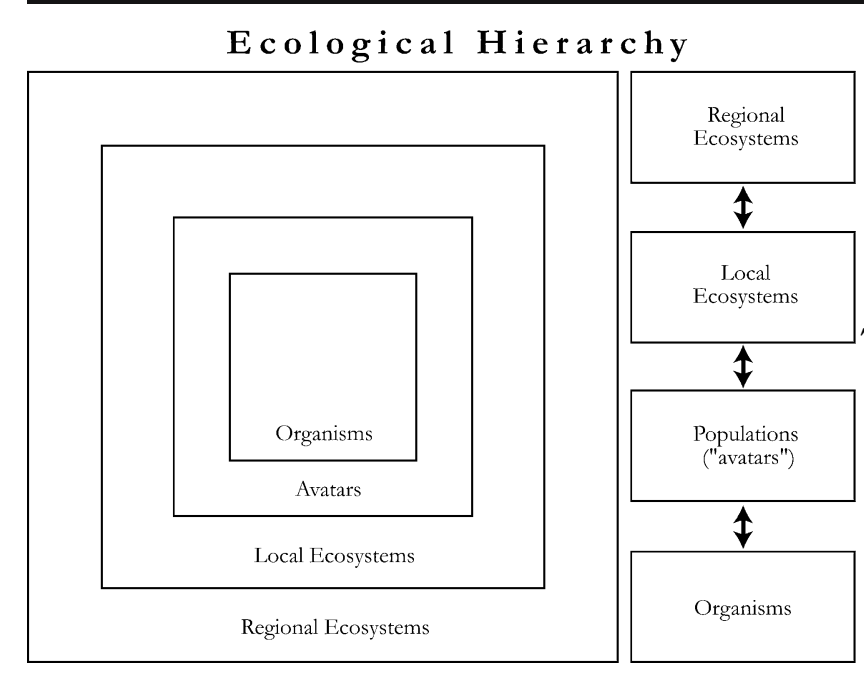

Fig. 2 The ecological hierarchy

If natural selection is the effect of relative economic success on reproductive success, we can also follow Darwin's definition of "sexual selection" (in the Descent of Man (Darwin 1871)): it is the "advantage organisms of the same sex within a species have over others purely in the ability to reproduce"-i.e., not a reflection of economic, but rather purely reproductive, success in its own right. For example, it is "natural selection" if the individuals in the deme who are most successful at foraging for food as a result leave more offspring to the next generation. It is "sexual selection" if better foraging (or any other economic activity) has nothing whatever to do with an individual's heightened success simply at the game of reproduction - of leaving more offspring to the next generation than other members of the deme.

\section{Hierarchies Applied: The Sloshing Bucket Model of Evolution}

The double-hierarchical structure of biological systems is based on the simple fact that organisms do two, and only two, kinds of things: they reproduce, and they procure matter and energy resources simply to live. However, whereas the description of this dual system enables us to see the fundamental nature of natural (and sexual) selection, these hierarchies seem rather abstract. Yet the difference between immediate, personal survival, and one's own sexual reproduction is clear enough. Taken on a grand scale, the difference between staying alive and generating new life may be viewed in a conceptual framework that tells us much. First, it explains how processes at all levels have interacted to produce the history of life that we see both in the fossil record, and the ecological and phylogenetic structure of life as it now exists on earth. And second,

mapping patterns of the actual evolutionary history of life on earth onto the hierarchy scheme yields a general vision of the evolutionary process (I call it the "sloshing bucket" (Eldredge 2003) for reasons explained below) that I further believe has profound implications for curriculum development at all educational levels.

Consider what happens when local ecosystems are disturbed —or eradicated altogether. R.G. Johnson (Johnson 1972) once studied the slow migration of a sandbar over the bottom of a portion of Tomales Bay in California. As the sandbar progressed, all the marine invertebrates on the bay's floor were exterminated. However, at the back edge of that sandbar, larval recruits virtually instantly were colonizing the newly reexposed normal sediments as the bar passed by. Where did they come from? Adjacent demes. Was evolution occurring? There is always molecular variation present, and perhaps some form of selection was indeed going on. But, in terms of the quickly rebuilt components of the normal Tomales Bay benthic ecosystem, recruitment from nearby demes quickly resulted in the reestablishment of the system that looked in all respects like it did before the sandbar passed over. Thus, from the standpoint of morphological adaptation, Johnson could detect no discernible evolutionary change.

Now consider the other extreme: global mass extinctions. The great mass extinctions of the geological past have radically altered the ensuing complexion of life on earth. Dinosaurs and mammals both arose in the midTriassic Period, some 215 million years or so ago. For reasons no one pretends to know, it was the dinosaurs (and collateral reptilian kin, such as pterosaurs and various groups of aquatic reptiles) that dominated the vertebrate component of terrestrial ecosystems. Ecologically speaking, the mammals, although diversified into several different major groups, were bit players throughout the Mesozoic.

\section{Ecological Hierarchy}

Biosphere

Regional

Ecosystems

Local

Ecosystems

Populations

("avatars")

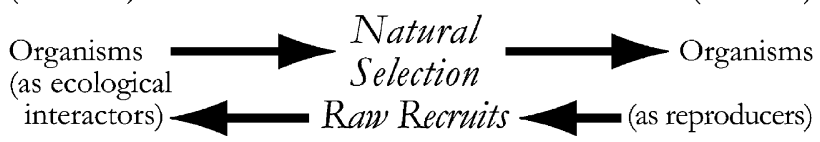

Fig. 3 The two hierarchies and natural selection

Evolutionary Hierarchy

Larger Groups of Species

Species Populations ("demes") 
For the most part quite small, they lived "in the interstices of the dinosaur's world" - to paraphrase the great mid-20th century paleontologist Alfred Sherwood Romer.

Several times (most notably at the end of the Triassic Period, and again at the end of the Jurassic Period) extinction thinned the ranks of dinosaur diversity, but each time the dinosaurs rediversified and maintained their dominant grip on Mesozoic terrestrial vertebrate life. This situation prevailed for nearly 150 million years - until the extraterrestrial impact(s) 65 million years ago eradicated what was left of the dinosaur fauna. Only then-albeit after a lag of some 5-7 million years-was it the surviving mammals who began to evolve rapidly. For the first time in their already-long history, there were mammals of all sizes (although none as large as the largest dinosaur!); there were omnivores, carnivores, herbivores, and scavengers. Some of them eventually learned to fly, whereas several other groups reinvaded the sea.

The point is that, whereas the reptilian dinosaurs were dominant, the mammals did not evolve into the impressive array of adaptive kinds we see in the Tertiary and see around us today (albeit in dwindling numbers as we are in the throes of the modern human-induced "sixth extinction"). The extinction of the dinosaurs effectively took the lid off, and the mammals, after a lag, responded with repeated bursts of evolution, extinction, and more evolution, reconfiguring the niches once occupied by their extinct predecessors.

The degradation of local ecosystems and its consequences, when juxtaposed with the evolutionary reactive effects of mass extinctions, suggests a general prediction about evolution: most evolution - and certainly the appearance of most new species, must lie somewhere between local ecosystem degradation, on one hand, and global mass extinction on the other-with its proportionately great effects on the histories - extinction and evolution-of larger ("higher") taxa.

The prediction is confirmed: the fossil record documents hundreds, possibly thousands, of "turnover" events, where environmental degradation was sufficiently severe that entire species began to succumb to extinction - and new species quickly evolved to populate the newly reconstituted environment. Paleontologist Elisabeth Vrba (Vrba 1985) (who coined the term "turnover") points to a spasm of global cooling that modified the wet woodland ecosystems of eastern and southern Africa beginning ca. 2.8 million years ago; in a relatively brief period of a few hundred thousand years, the woodlands were replaced by drier open savannah grasslands-forcing many species either to migrate out, or suffer extinction. Meanwhile, species already adapted to grasslands migrated in-and new grassland-adapted species quickly evolved from some of the surviving remnants of the previous ecological regime.

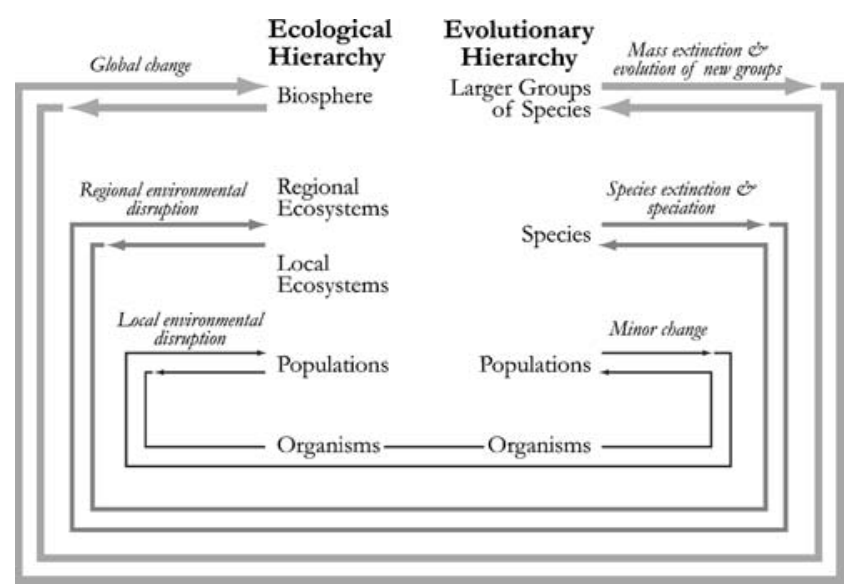

Fig. 4 The sloshing bucket theory of evolution

And, of course, the species who were equally well adapted to wet woodlands and open savannahs (impalas are an example) managed to survive unchanged.

Hence the "sloshing bucket" (Fig. 4): nothing much happens in terms of discernible morphological evolution until environmental change overturns the ecological applecart. A little degradation - including the deaths of individuals - will not lead to measurable evolutionary change. A huge amount of devastation, on the other hand, can lead to the extinction of entire major groups-prompting the evolution of other large-scale groups. And on intermediate spatiotemporal scales - where individual species are driven to extinction, but not entire higher taxa-new species evolve, populating the new ecosystems. Van Dam and colleagues (Van Dam et al. 2006) have recently shown such turnovers to be virtually the entire basis of rodent evolution as preserved in the fossil record of Spain over the past 22 million years.

The greater the magnitude of the environmental event, the greater the change in ecosystems, including the magnitude of diversity loss through extinction; the greater the loss of higher taxa, the more different will be the newly evolved taxa, and thus the nature of the succeeding ecosystems that replaced the prior disturbed systems. Thinking of our diagram, it is like water sloshing in the bucket - the size of the sloshes depending on how hard the bucket is jolted. Thus, Fig. 4 depicts a real-life "fleshing out" of the abstract dual hierarchy structure by inserting actual patterns of the history of life - the "sloshing bucket."

\section{Implications for Curriculum Development}

Not being an educator, I must leave it to professionals to see the full implications of the "sloshing bucket" for teaching evolution in the classroom. However, some of its 
virtues in this respect are obvious. For one thing, all of the disciplines in biology (and geology and environmental sciences) that have the slightest bearing on evolution are revealed at a glance. Teachers can discuss ecology with the promise that its connections to evolution will, in the course of study, be made abundantly clear. Likewise for paleontology, systematics - and all forms of organismal biology. That hierarchical thinking applies to the genome has also recently been made clear (Gregory 2004). At the very least, hierarchies-along with its fleshed out version as the "sloshing bucket" - can potentially serve as a useful heuristic structure for teaching evolution at all educational levels.

Acknowledgments This paper is based on a presentation in the Hierarchy Symposium at the Genoa Science Festival, November, 2006. I thank the Festival organizers Vittorio Bo and Telmo Pievani for their support to the Hierarchy Research Group's discussions and public presentations at that meeting.

\section{References}

Damuth J. Selection among "species": A formulation in terms of natural functional units. Evolution 1985;39:1132-46.

Darwin C. The descent of man, and selection in relation to sex. London: John Murray; 1871.

Eldredge N. The sloshing bucket: How the physical realm controls evolution. In: Crutchfield J, Schuster P, editors. Evolutionary dynamics. Exploring the interplay of selection, accident, neutrality, and function. SFI studies in the sciences of complexity series. New York: Oxford University Press; 2003. pp. 3-32.

Gregory TR. Macroevolution, hierarchy theory, and the C-value enigma. Paleobiology 2004;30(2):179-202.

Johnson RG. Conceptual models of benthic marine communities. In: Schopf TJM, editors. Models in paleobiology. San Francisco: Freeman, Cooper; 1972. pp. 148-59.

van Dam JA et al. Long-period astronomical forcing of mammalian turnovers. Nature 2006;443:687-691. DOI 10.1038/nature05163.

Vrba ES. Environment and evolution: alternative causes of the temporal distribution of evolutionary events. S Afr J Sci 1985;81:229-36. 